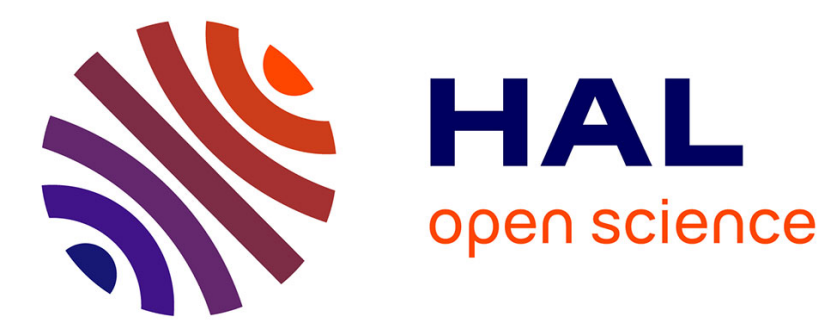

\title{
Threshold-based routing algorithm for RF-NoC OFDMA architecture
}

\author{
Habiba Lahdhiri, Jordane Lorandel, Emmanuelle Bourdel
}

\section{To cite this version:}

Habiba Lahdhiri, Jordane Lorandel, Emmanuelle Bourdel. Threshold-based routing algorithm for RF-NoC OFDMA architecture. ReCoSoC 2019, Jul 2019, York, United Kingdom. hal-02433969

\section{HAL Id: hal-02433969 \\ https://hal.science/hal-02433969}

Submitted on 9 Jan 2020

HAL is a multi-disciplinary open access archive for the deposit and dissemination of scientific research documents, whether they are published or not. The documents may come from teaching and research institutions in France or abroad, or from public or private research centers.
L'archive ouverte pluridisciplinaire HAL, est destinée au dépôt et à la diffusion de documents scientifiques de niveau recherche, publiés ou non, émanant des établissements d'enseignement et de recherche français ou étrangers, des laboratoires publics ou privés. 


\title{
Threshold-based routing algorithm for RF-NoC OFDMA architecture
}

\author{
Habiba Lahdhiri, Jordane Lorandel and Emmanuelle Bourdel \\ Laboratoire ETIS, Université Paris-Seine, Université de Cergy-Pontoise, ENSEA, CNRS \\ F-95000 Cergy, France. \\ \{habiba.lahdhiri, jordane.lorandel, emmanuelle.bourdel\}@ensea.fr
}

\begin{abstract}
Network-on-chip (NoC) is currently the chosen paradigm for interconnection in multicore architectures. On the other hand, the development of these architectures with thousands of cores leads to several issues regarding latency and power consumption, especially with conventional wired interconnects. Moreover, they do not support specific features like broadcast and multicast. Radio-Frequency NoC based on OFDMA seems an appropriate solution to overcome such issues, providing low latency for long communications and a significant communication bandwidth with a high spectral efficiency. It also permits a dynamic allocation of the available bandwidth among the different elements of the NoC, according to the communication needs. The routing algorithm has a significant impact on the overall performance of a RF-NoC, by diffusing the traffic in a suitable way between the wired and the wireless paths in order to improve the throughput and reduce the latency. In this paper, a threshold-based routing algorithm is proposed and evaluated for a $32 \times 32 \mathrm{RF}-\mathrm{NoC}$ OFDMA architecture with 32 concurrent wireless channels. We demonstrate the importance to finely tune the threshold parameter to achieve a certain level of performance. Simulation results show a significant improvement in terms of latency and throughput.
\end{abstract}

Index Terms-RF; NoC; OFDMA; Routing; Reconfigurable;

\section{INTRODUCTION}

The development of new architectures with thousands of cores on the same chip, communicating together, reveals new challenges. In particular, communications between the cores using conventional wires create a bottleneck, leading to poor performance and suffering from a high latency. This is particularly true when long communications across the chip occurs. Several interconnect technologies have been proposed since the last decades, permitting to overcome the main issues of classical wired NoC.

Firstly, optical technology is a promising alternative to conventional NoC, providing shortcuts for destination and source that are faraway from each other. The use of optical interconnect allows to achieve high data transmission rate and a very low latency. A critical limitation is the need for an external light source. 3D-NoC is an attractive approach to solve interconnect scaling. This architecture fulfils the demands for future multicore architectures, exploiting the short vertical links between adjacent layers that significantly improves the network performance [1]. A Multi-layer NoC architecture reduces the distance between routers in a considerable way. In 3D-NoC energy reduction can be achieved up to $29 \%$, in the average packet latency up to $2 \%$ and in the area occupied by the routers of the $\mathrm{NoC}$ up to $18 \%$ [1]. However, the advantages of this technology cannot overlook thermal issues when the number of layers increases. Some proposed architectures benefit from both 3D and optical technologies [2]. The upper layer contains the optical data transmission network, which is responsible for optical payload packets transmission. The bottom layer contains an electronic control network used to route control packets and to configure the optical network. Power analysis based on synthesis results shows that the optical NoC can have a $70 \%$ power saving for 2048B packets.

$\mathrm{RF}$ interconnects can also be used for long-range communications. RF interconnects add a set of shortcuts to the network that significantly reduces the latency. Moreover, due to the reconfigurability of RF interconnections, it is possible to modify the bandwidth allocation and adapt it to the demand. RF waves can be transmitted through a wave-guide or an antenna. Solutions using antennas have greater flexibility, but they also increase consumption in comparison to wave-guides, and are less robust against interferences. Another solution of interconnect is the combination of optical and wireless Network OWN [3]. The communications within the same cluster (64 cores) are realised with one-hop photonic interconnect while communications between clusters use one-hop wireless interconnect. Such architecture enhances scalability and simulation results show that the energy/bit for OWN is $30.36 \%$ lower than wireless and $13.99 \%$ higher than photonic architecture. Several studies [4], [5] have made preliminary comparisons among these different interconnect technologies. Table I gives an overview of the main advantages and limitations of the interconnect technologies mentioned previously.

To fully exploit an interconnect technology as optical or $\mathrm{RF}$, new efficient communication mechanisms have to be proposed. In particular, all these interconnect technologies require a multiple access technique to efficiently share the spectrum resources between the numerous elements wishing to communicate. In addition, resource allocation has to be fairly allocated among the elements of the network [6].

A lot of multiple access techniques exists in the literature, such as Frequency Division Multiple Access (FDMA), Time-Division Multiple Access (TDMA), Wavelengh Division Multiple Access (WDMA). Multi-carriers modulation tech- 
TABLE I

COMPARISON OF INTERCONNECT TECHNOLOGIES

\begin{tabular}{|l|l|l|l|l|}
\hline & 3D-NoC & 0-NoC & \multicolumn{2}{|c|}{ RF-NoC } \\
\cline { 2 - 5 } & Multiple layers stacks & $\begin{array}{l}\text { Silicon photonic compo- } \\
\text { nents }\end{array}$ & Wave-guide & On-chip antenna \\
\hline Mardware Requirements & $\begin{array}{l}\text { Higher connectivity } \\
\text { shorter path }\end{array}$ & $\begin{array}{l}\text { High speed optical devices } \\
\text { and links, low latency }\end{array}$ & low latency / dynamic bandwidth allocation & [4], [7] \\
\hline Power dissipation issue & $\begin{array}{l}\text { Shorter average path } \\
\text { length }\end{array}$ & $\begin{array}{l}\text { Negligible power dissipa- } \\
\text { tion in optical data trans- } \\
\text { port }\end{array}$ & Multi-hop paths replaced by single hop links & [5], [8] \\
\hline Challenges & $\begin{array}{l}\text { thermal issue because of } \\
\text { high-power density }\end{array}$ & $\begin{array}{l}\text { Integration of on-chip } \\
\text { photonic components }\end{array}$ & $\begin{array}{l}\text { mixed-signal } \\
\text { physical design } \\
\text { problem }\end{array}$ & $\begin{array}{l}\text { integration of on- } \\
\text { chip antennas. }\end{array}$ \\
\hline
\end{tabular}

niques such as Orthogonal Frequency Division Multiplexing (OFDM), achieve a high spectral efficiency while transmitting data among multiple orthogonal sub-carriers. OFDM provides many advantages such as robustness against inter-carrier interference, high spectral efficiency, simple equalisation in the frequency domain at the receiver side, etc. However, OFDM is very sensitive to synchronisation issues. Orthogonal Frequency Division Multiple Access (OFDMA) is based on OFDM allowing multiple users to share a common bandwidth easily. From a design point of view, OFDMA needs fewer hardware resources for the RF transceiver as compared to FDMA, which necessitates as many local oscillators as the number of elements connected to the interconnect. OFDMA is thus a more scalable technique. Moreover, sub-carriers allocation can be directly done from the base-band and dynamically changed while the application is running, allowing the frequency bandwidth used by each core or group of cores to be adapted according to their needs [10]. For the aforementioned reasons, OFDMA was chosen as the most well-suited technique in our study.

A key element for NoC is the routing algorithm. To keep increasing NoC performance implies the development of new routing algorithm that can fully exploit the interconnect capabilities. In this paper, a routing algorithm for OFDMAbased RF-Noc is proposed. It allows to distribute the traffic communication by comparing the distance between source and destination to a user-defined threshold. From our knowledge, the algorithm has already been proposed in [11] but was never applied to an OFDMA RF-NoC, combining RF-waveguide and wired NoC. Moreover, the choice of threshold was not deeply investigated. A deep study is thus provided to deeply investigate the choice of threshold depending on the target latency and throughput.

This paper is organised as follows. First, Section II details the new interconnect technologies used in multicore architectures and their associated topologies and routing algorithms. Then, the RF-NoC architecture based on OFDMA is presented in Section III and the proposed routing algorithm is given in Section IV. Simulation results are presented in Section V and finally conclusion and future works are described in Section VI.

\section{BACKGROUND AND RELATED WORKS}

\section{A. Topology}

The topology defines the physical layout and the connections between nodes in the network. The topology impacts network cost and performance. It influences the latency by determining the number of hops a packet must perform from the source to its destination.

For conventional NoCs built around a wired interconnect between nodes, topologies can be divided into 3 categories: direct, indirect and irregular. Direct topologies refer to meshes or tori. Indirect topologies refer to butterflies networks. Finally, irregular topologies refer to multiprocessor system-on-chip (MPSoC) with a large range of heterogeneous intellectual property (IP) blocks.

To benefit from new interconnect technologies, novel topologies have been proposed. For example, WiNoC use the wireless interconnect between nodes to reduce the network latency, especially for these long-distance nodes [12].

In order to provide scalability and flexibility, the wireless links are used to replace the wired interconnect. In a purely wireless $\mathrm{NoC}$, all the links between two nodes should be wireless. As the number of nodes is growing, wireless channels cannot handle all the links of simultaneous communications. Thus, purely wireless NoC topologies scale poorly. To overcome this issue a multi-channel WiNoC was proposed [13]. The network is composed of RF nodes working as a wireless router with ultra-wide-band (UWB) transceivers and antenna. The wired links in the conventional 2D mesh are replaced by high-bandwidth RF links.

Another topology, called hybrid, is based on wired links for short-distance communications and wireless/optical links for long-distance communications. Hybrid topology is constructed by replacing some of the routers in the $2 \mathrm{D}$ mesh-based topology with wireless routers, generally one wireless router per cluster (sub-net) which have both wired and wireless links [14]. An example of hybrid topology is the multiple tiers hybrid topology proposed to deal with scalability problems [15]. The particularity of this topology is the recursive wireless interconnection structure named WCube which is also a hybrid topology with a multi-tier structure, including the wireless 
backbone and wired edges. The wired edge (CMesh), which is a 2D mesh with 4-way concentration (concentration in on-chip networks is to share the router among different (processors) nodes). Another example is the Small-world-based topology which divides the whole network into multiple small groups (sub-nets) with different heterogeneous topologies. It can be any of the conventional NoC topologies, such as mesh, tree, ring etc. The upper level of the network contains hubs connected by both wired and wireless links [5].

Irregular NoC topologies are usually used for MPSoCs with heterogeneous IP blocks. In [16] a low cost and efficient distributed minimal table-based routing for an irregular WiNoC topology is presented. With these particular topologies, the routing algorithm is very important to fully exploit the capacity of the additional interconnect, conditioning NoC performance.

\section{B. Routing Algorithm}

The routing algorithm defines the way to route packets from source to destination. It must distribute the traffic in an appropriate manner, avoiding deadlocks and significant delays. An efficient routing algorithm allows thus the improvement of the network throughput and the reduction of the latency. In conventional wired NoCs, routing algorithms can be classified into two categories: deterministic and adaptive. Deterministic routing algorithms route packets from point $\mathrm{A}$ to another point B along a fixed path and offer the best choice for uniform or regular traffic patterns. Adaptive routing algorithms use information about the network traffic and channel conditions to avoid congested network regions. An adaptive approach is preferable when dealing with irregular traffic or in networks with unreliable nodes and links.

For RF-NoC architecture, the routing algorithm is specific to the topology. As an example, Location-based algorithm (LBR) has been proposed in for McWiNoC [13]. It is based on the knowledge of the router node and all its neighbours to make the routing decision. When routing a packet towards the destination, it will choose to send the packet to its neighbour which is geographically closest to the destination to the destination. In order to optimise the LBR algorithm, the authors divided the chip area into four quadrants from the perspective of a node. When a router receives a packet, by checking the position of destination $\mathrm{D}$, only one quadrant containing node $\mathrm{D}$ will be chosen. Then, only the neighbours of the selected quadrant will be calculated for the shortest path.

In [15], a hybrid WiNoC is divided into 3 tiers. For each tier, a routing algorithm must be chosen first. Then, further routing strategies are required to decide if and how packets are transmitted between the different layers. The authors devise new two-tier wormhole based routing algorithms that are deadlock free and ensure a minimum latency route on a 1000core on-chip interconnect network, showing a reduction of the observed latency going from $20 \%$ up to $45 \%$.

In Small-world-based routing [5], the routing within the cluster can be any of the traditional NoC routing algorithms according to the topology of each sub-net, for example XY routing algorithm. For the inter cluster transmission, the packet will be routed through the hubs. Therefore, whether using the wireless links or just taking the wired link along the ring is very important to the network performance.

Adaptive routing is based on the same concept as the previous algorithms by using a traditional NoC routing algorithm within the cluster (wired sub-net). However, for communications between different clusters an adaptive routing algorithm is needed. For example, in [14] the path selection is a function of travelling distance and buffer utilisation status to dynamically avoid the congestion.

Finally, for irregular WiNoC topologies, traditional routing algorithms may not work in some situations. Therefore $\mathrm{Wu}$ et al. [17] proposed a distributed minimal table based routing scheme by utilising the geographic distance information. The proposed routing can be also applied to the conventional irregular mesh topology connected by a wired network.

RF-NoC OFDMA topology combines both wired mesh topology and radio hubs for RF interconnections. For this specific architecture a dedicated routing algorithm is required which will be presented instead.

As the number of RF interfaces in the same chip is growing, an efficient medium access control (MAC) strategy is required for every RF-NoC architecture to efficiently share the RF medium. The MAC is a major factor of performance in WiNocs since it forbids simultaneous accesses to the same channel.

Related works on MAC for WNoCs can be classified into two types. Contention free [18] approaches are based on the use of a coordinated access protocol. The most common example is the token-ring, allowing only the RF interface holding the token to access the medium and transmit data. This group of MAC strategies has the advantages of delivering a high throughput and avoiding collisions, but does not work well under variable workloads since bandwidth is statically allocated. An other limitation is the overhead related to the token circulation that increases with the number of RF interfaces.

The second type of MAC strategy is called Contention based. This group is specified by the random access that means all nodes can try to transmit on the shared medium at any time instant, offering flexibility and reducing the latency. However, the risk of collision is increased, impacting negatively the throughput of the network [19].

For our study, we selected a hybrid topology for the RFNoC that uses the RF link for long range communication. To share the common bandwidth, Orthogonal Frequency Division Multiple Access (OFDMA) was chosen. Finally, for the routing algorithm, we adapted the algorithm proposed in [14] for the RF-NoC OFDMA architecture detailed in the next section.

\section{RF-NOC ARCHITECTURE WITH OFDMA}

The studied RF-NoC architecture, illustrated in Figure 1, contains two levels of hierarchy: clusters and cores. At each 
hierarchical level corresponds a specific interconnect, respectively RF interconnect and wired NoC. Each cluster contains 32 routers (1 per core), which are interconnected with a wired NoC while the communications between clusters are realised through the RF wave-guide. Note that communications between clusters are also possible through the wired interconnect. Finally, the global RF-NoC architecture integrates 1024 cores (and routers).

Each cluster features an RF-NoC interface consisting in one OFDMA transmitter and one receiver, called radio hub [20]. The RF-NoC interface is connected on one side to the four central routers as illustrated in Figure 1, and on the other side to the RF wave-guide.

Accessing the wave-guide is thus possible using Orthogonal Frequency-Division Multiple Access (OFDMA) that achieves a high spectral efficiency by dividing the bandwidth into several orthogonal narrow sub-channels. The use of OFDMA allows simultaneous communications between several radio radio using different frequency channels.

In our configuration, a bandwidth $B$ of $20 \mathrm{GHz}$ is divided into 1024 sub-carriers. A frequency spacing of $19.53 \mathrm{MHz}$ between each sub-carrier is thus obtained. The OFDMA symbol duration of $T_{s}$ is computed as follows and is equal to $51.2 \mathrm{~ns}$ :

$$
T_{s}=(1 / B) * N_{s c}
$$

with $N_{s c}$ representing the number of sub-carriers.

The data rate $R$ can be changed to transmit more information per OFDMA symbol, by modifying the symbol modulation:

$$
R=\left(M * N_{s c}\right) / T_{s}
$$

where $M$ is the modulation order, representing the number of bits per symbol (e.g. 2 for QPSK, 4 for 16-QAM, etc.).

Each cluster can transmit data through its RF interface using a group 32 contiguous sub-carriers, but can receive the entire bandwidth, making possible multicast and broadcast communications between clusters.
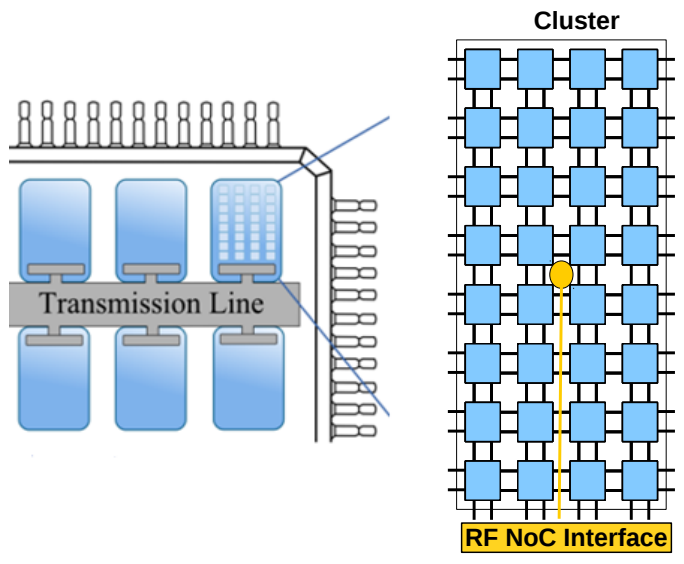

Fig. 1. RF-NoC architecture
Throughout the choices of MAC technique and topology, such RF-NoC architecture ensures scalability while being physically compatible with existing CMOS technology. However, to efficiently exploit the RF-NoC architecture, a main issue is the definition of an appropriate routing algorithm.

\section{Threshold-BASED Routing Algorithm}

The routing algorithm decides which path a packet will take from the source to its destination. It is responsible for diffusing the traffic on the network in a suitable way to improve the throughput of the network and reduce the latency.

In fact, the algorithm is based on the calculation of the distances (number of hops) from the packets source node $S$ to its destination node $D$ using the wireless link and the wired $\mathrm{NoC}$, as shown in eq. 3 and eq. 4. If the wireless distance is greater than the wired distance then the packets are transmitted using the wireless link, leading to a reduction of the average delay of the network. A naive solution could be to take the minimum distance but this could rapidly create a bottleneck at the radio hub. Therefore, an adjusting threshold $\gamma$ is defined to control the utilisation of the RF link to avoid network congestion.

$$
\begin{gathered}
D_{\text {wired }}=\left|\left(x_{s}-x_{d}\right)+\left(y_{s}-y_{d}\right)\right| \\
D_{\text {wireless }}=\left|\left(x_{s}-x_{c s}\right)+\left(y_{s}-y_{c s}\right)\right|+\left|\left(x_{d}-x_{c d}\right)+\left(y_{d}-y_{c d}\right)\right|
\end{gathered}
$$

The threshold mainly depends on the network size and in our study, an optimum value of $\gamma$ has been determined and explained later. To illustrate the routing algorithm, we present three cases. Firstly, if the source $S$ and the destination $D$ belong to the same cluster, there is no need to use wireless links. The packet is transmitted through the wired links according to a XY routing. Secondly, if the $S$ and $D$ are not from the same cluster but they are just close enough, satisfying $D_{\text {wired }}+\gamma<D_{\text {wireless }}$, then packets are routed using the wired NoC. Thirdly, only for long distance communications, where $D_{\text {wired }}+\gamma>D_{\text {wireless }}$, the routing decision is to take the wireless path by reaching the closest router attached to the radio hub in the cluster of $S$ using XY routing algorithm. The packet is then transmitted using the wireless link and is delivered to the closest router from the destination that is attached to the radio hub. Finally, if needed, a XY routing is used to route the packet towards the destination router.

To avoid deadlock situations, virtual channels (VCs) are used. Two different sets of VCs are deployed in each router and each radio hub. The first set of VCs is used for communications going from the source to CS using the wired path. The second set of VCs is dedicated to packets going from the source to the destination using wired path or packets going from CD to the destination. As shown in [11], VCs ensure the deadlock free property because they separate the allocation of physical channels from buffers, so that the physical channels are given to another packet when a packet is blocked.

The Threshold-Based Routing (TBR) algorithm is summarised in algorithm 1. 


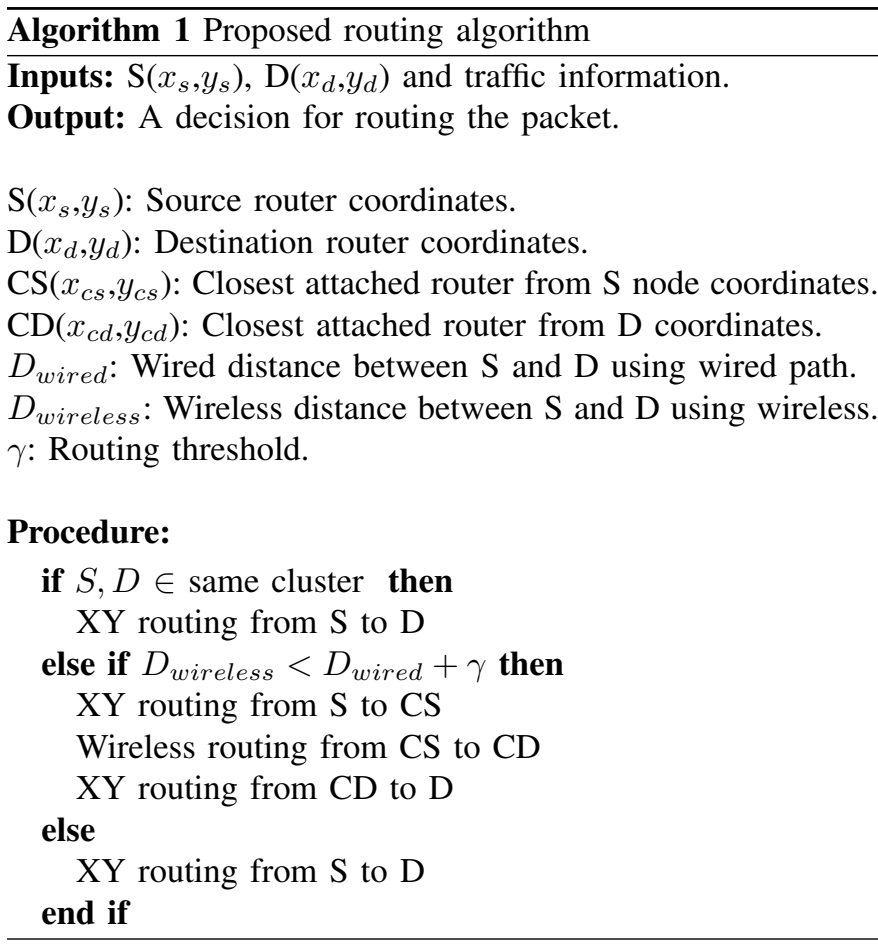

\section{Simulation Results}

To evaluate the performance of the routing algorithm for our 1024-core RF-NoC OFDMA architecture, the Noxim simulator was used [21]. It is an existing SystemC-based NoC simulator, allowing to perform cycle-accurate simulations. It supports the simulation of both conventional wired and wireless NoC architectures and several topologies, while including different routing algorithms, e.g. XY, Odd-Even, West-first, North-last, and many traffic distributions. By default, a tokenring technique is used to specify how to access the radio channels, and only one radio hub can transmit information on the wireless link at a time. However, Noxim was extended to support OFDMA and concurrent accesses to wireless channels. The TBR routing algorithm described in Section IV was also integrated into the simulator.

Table III indicates the simulation parameters used to evaluate the architecture and the performance of the TBR routing algorithm.

TABLE II

SiMULATION NOC PARAMETERS

\begin{tabular}{|l|l|}
\hline Parameter & Value \\
\hline Clock frequency & $1 \mathrm{GHz}$ \\
Number of cores on chip & $1024(32 * 32)$ \\
Number of radio-hubs \& clusters & 32 \\
Number of radio channels & 32 \\
Number of virtual channels & 2 \\
Packet size [flit] & 8 \\
Flit size [bit] & 64 \\
Bandwidth & $20 \mathrm{GHz}$ \\
OFDMA sub-carriers & 1024 \\
Modulation order & $16-\mathrm{QAM}$ \\
Data rate & $80 \mathrm{Gbps}$ \\
Simulation Time (cycle) & 50.000 \\
\hline
\end{tabular}

We first evaluate the average latency of the architecture, corresponding to the time spent by a packet to cross the network from the source to the destination node. Figure 2 gives the average delay versus the threshold value for TBR and conventional XY routing algorithm, respectively illustrated with the blue and red bars. The simulations are done with a constant value of Packet Injection Rate (PIR) of 5E-3 packet/cycle/node. It can be observed that the average delay is very important for TBR when the threshold is equal to zero, giving an average use of the RF link about $96 \%$, as indicated in Table III. When the threshold increases, we notice that the latency gets reduced in a significant way until it reaches an optimal value for a threshold of 20 .

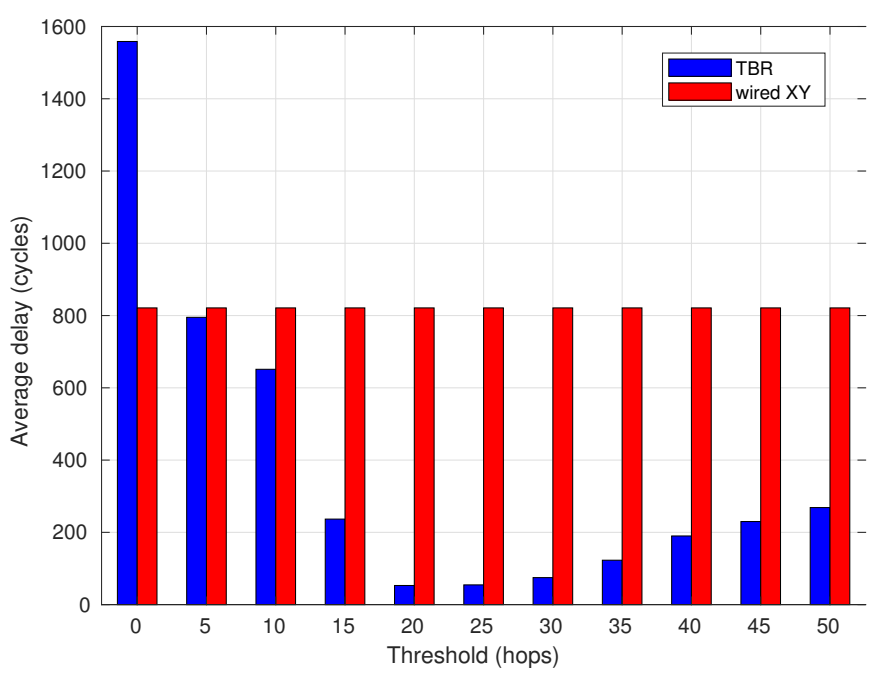

Fig. 2. Average delay vs threshold for TBR and XY routing algorithms

TABLE III

RF UTILISATION AND DELAY FOR TBR ALGORITHM

\begin{tabular}{|l|l|l|}
\hline Threshold & $\begin{array}{l}\text { Average delay } \\
\text { (cycles) }\end{array}$ & $\begin{array}{l}\text { Average RF } \\
\text { utilisation (\%) }\end{array}$ \\
\hline 0 & 1558 & $96 \%$ \\
5 & 795 & $87 \%$ \\
10 & 651 & $71 \%$ \\
15 & 236 & $56 \%$ \\
20 & 52 & $41 \%$ \\
25 & 54 & $28 \%$ \\
30 & 74 & $18 \%$ \\
35 & 122 & $13 \%$ \\
40 & 190 & $10 \%$ \\
45 & 229 & $9 \%$ \\
50 & 268 & $8 \%$ \\
\hline
\end{tabular}

When $\gamma$ is 20, the latency is reduced by $\mathbf{9 3 \%}$ as compared 
to the conventional XY routing. Referring to the RF-NoC architecture, this particular threshold value corresponds to a communications with a distance of at least two clusters, indicating that the RF link has to be used for long communications only. However, once the threshold is greater than the optimal value, the average delay increases again, but it is still lower than for XY routing, highlighting the benefit of integrating RF interconnect in addition to a wired NoC. Finally, the choice of the threshold value has a direct impact on the network latency while depending of the RF-NoC size. For our 1024-core RFNoC OFDMA architecture, the optimal threshold value is $\gamma=20$. This value will be used as reference in the rest of the paper.

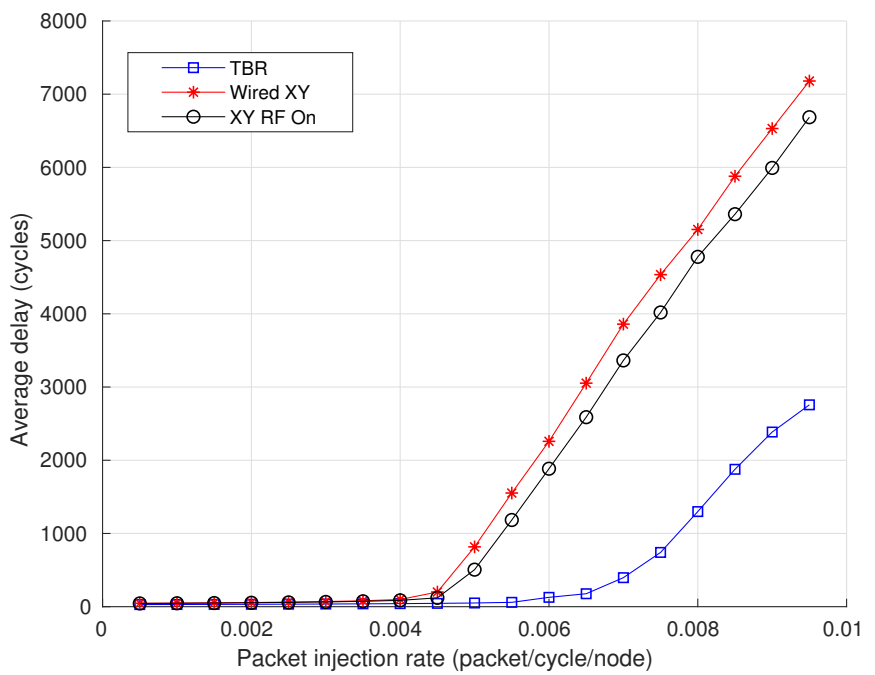

Fig. 3. Average delay vs PIR under random traffic

Figure 3 gives the average delay against PIR for TBR, XY with RF path and XY without RF. By comparing the red and black curves is demonstrated the importance of providing an appropriate routing algorithm to efficiently exploit the RF interconnect. As illustrated, the red curve representing a wired $\mathrm{XY}$ routing suffers from a slightly higher latency as compared when RF communications are activated. TBR achieves a lower average latency when the PIR is getting higher than 4E-3. A reduction of $95 \%$ of the latency is denoted as compared to wired XY routing algorithm at PIR $=6 \mathrm{E}-3$. We can note that there is no significant gain in terms of latency to use RF communications at low PIR.

Then, the performance of the TBR algorithm is compared to existing routing algorithms, i.e. North Last, Odd-Even and West-First, under a random traffic. Figure 4 gives the evolution of the network latency for several values of PIR. Recall that the threshold value was fixed at 20 hops for TBR algorithm. As it can be noticed, TBR provides the lowest latency at any PIR value in the range [5E-4,1E-2], but the gap is remarkable from a PIR of $4 \mathrm{E}-3$. The average wireless utilisation for PIR $=6 \mathrm{E}-3$ is equal to $35 \%$. The optimal value of threshold has prevented congestion of radio hubs and delayed network saturation that's way TBR provides better performance.

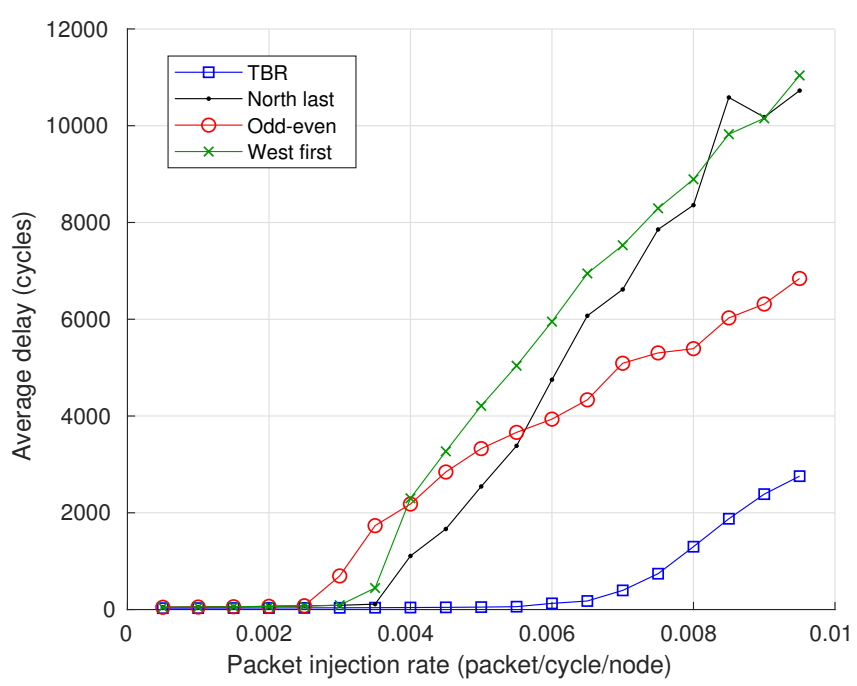

Fig. 4. Average delay vs PIR for several routing algorithms under Random traffic

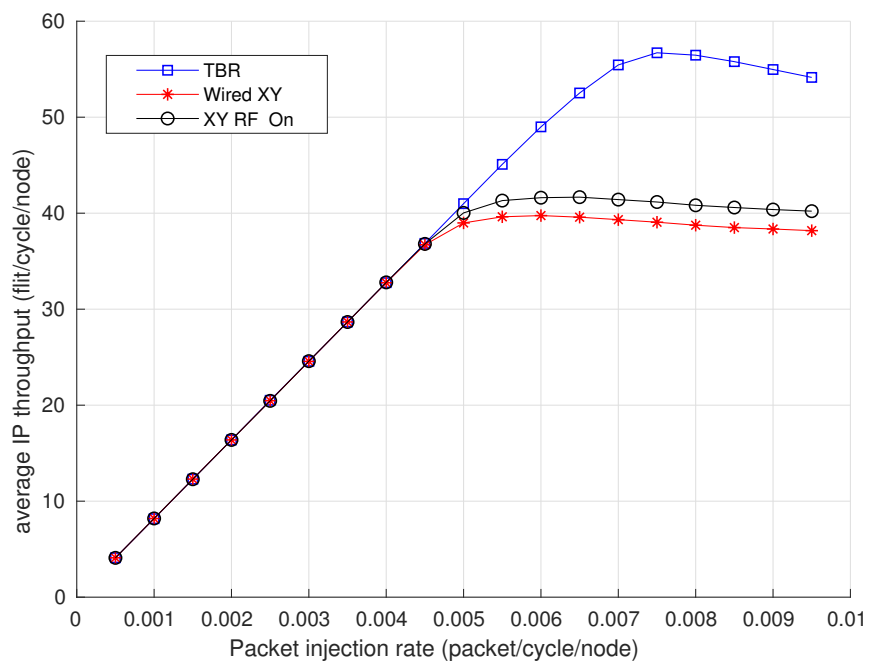

Fig. 5. Average IP throughput vs PIR under random traffic

The IP throughput was also evaluated for different values of PIR for the routing algorithms. As illustrated by Figure 5, threshold-based routing algorithm makes the network able to support a more important IP throughput, for PIR values greater than 5E-3. At PIR of 75E-4, an improvement of the IP throughput of $28 \%$ is achieved for TBR in comparison to XY routing with wireless links. For lower PIR values, the same IP throughput is achieved for the 3 cases studied.

The Performance of the TBR algorithm was also evaluated for another traffic pattern, Transpose 1, which provides more unbalance to the network, showing more differences between routing algorithms [22]. As illustrated by Figures 6 and 7 TBR achieves better performance in terms of latency and throughput. The first observation is the similarity of the two curves wired XY and XY with the RF link, which shows that such is our architecture (only the four routers in the center 


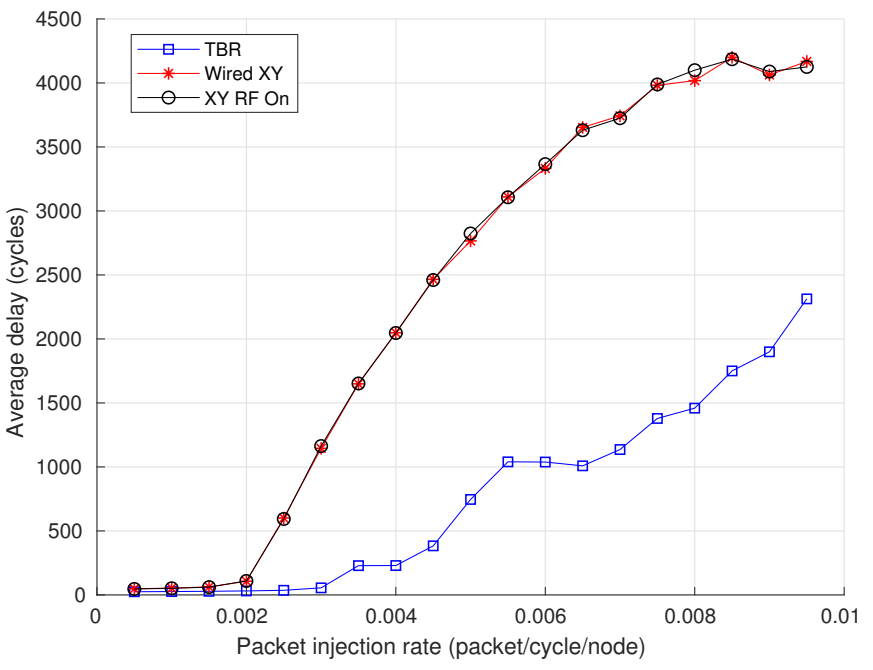

Fig. 6. Average delay obtained for Transpose 1 traffic

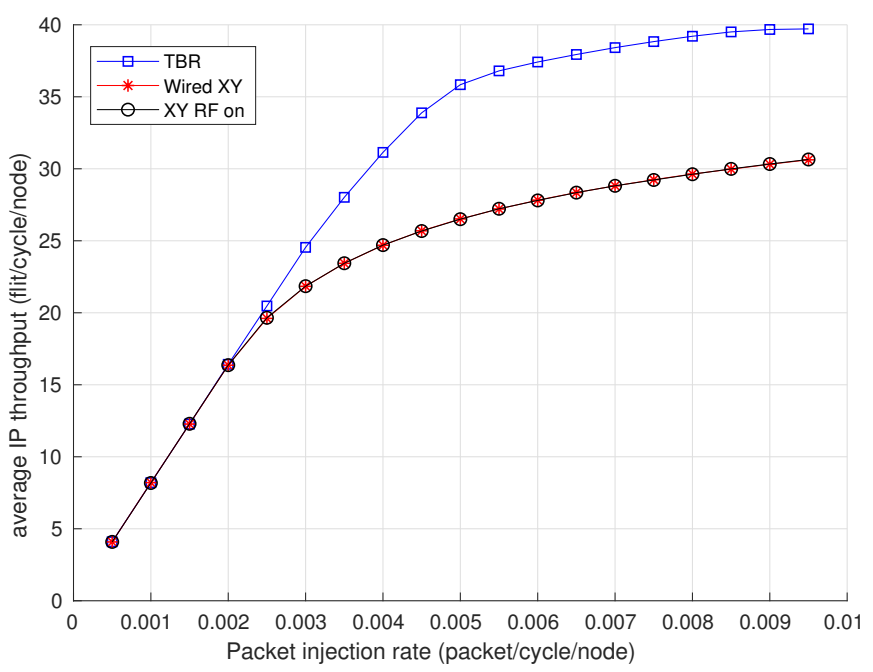

Fig. 7. Average IP throughput obtained for Transpose 1 traffic

of the cluster that are attached to the radio hub) and with this kind of traffic the RF link does not bring any gains in terms of latency and throughput. However, a reduction of $70 \%$ of the average latency is obtained for TBR at a PIR of $6 \mathrm{E}-3$ while the throughput is increased of around $27 \%$.

Throughout these results, TBR routing algorithm provides an efficient way of distributing the traffic on both wired and RF links, reducing the network latency and increasing the IP throughput for different traffic patterns. However, the choice of threshold is a critical step conditioning a certain level of performance. Finally, RF communications supporting by our 1014-core RF-NoC OFDMA architecture should only be used for long communication with the right threshold value to achieve better performance.

\section{CONCLUSiOn AND Future WORK}

In this paper, a threshold-based routing algorithm is evaluated for a 1024-core RF-NoC OFDMA architecture. We demonstrated the benefit of using RF-NoC for long communications and the impact of the threshold value on critical metrics such as latency and throughput, for different traffic patterns. The routing algorithm TBR is a preliminary step towards the development of a completely distributed cognitive RF-NoC based on OFDMA. Future work will focus on the development of a spectrum sensing technique into the RFNoC architecture, allowing to determinate spectrum access opportunities and dynamically allocate spectrum resources ondemand. The proposed architecture will also be evaluated using real traffic patterns to better investigate the behaviour of the network in these conditions.

\section{ACKNOWLEDGMENT}

The authors would like to thank M. Palesi and S. Monteleone for their support during the extension of Noxim.

This work is supported by a public grant overseen by the French National Research Agency (ANR) as part of the Investissements d'Avenir program (reference: ANR-16-IDEX0008).

\section{REFERENCES}

[1] T. T. Ye, L. Benini and G. De Micheli, "Analysis of power consumption on switch fabrics in network routers," Proceedings 2002 Design Automation Conference (IEEE Cat. No.02CH37324), New Orleans, LA, USA, 2002, pp. 524-529.

[2] Y. Ye, L. Duan, J. Xu, J. Ouyang, M. K. Hung and Y. Xie, "3D optical networks-on-chip (NoC) for multiprocessor systems-on-chip (MPSoC)," 2009 IEEE International Conference on 3D System Integration, San Francisco, CA, 2009, pp. 1-6.

[3] M. A. I. Sikder, A. K. Kodi, M. Kennedy, S. Kaya and A. Louri, "OWN: Optical and Wireless Network-on-Chip for Kilo-core Architectures," 2015 IEEE 23rd Annual Symposium on High-Performance Interconnects, Santa Clara, CA, 2015, pp. 44-51.

[4] L. P. Carloni, P. Pande and Y. Xie, "Networks-on-chip in emerging interconnect paradigms: Advantages and challenges," 2009 3rd ACM/IEEE International Symposium on Networks-on-Chip, San Diego, CA, 2009, pp. 93-102.

[5] S. Wang and T. Jin, "Wireless network-on-chip: a survey," in The Journal of Engineering, vol. 2014, no. 3, pp. 98-104, 32014.

[6] J. Luo and al., "Channel Allocation protocol for reconfigurable Optical Network-on-Chip," in Workshop on Exploiting Silicon Photonics for Energy-Efficient High Performance Computing, 2015.

[7] A. B. Ahmed, A. B. Abdallah and K. Kuroda, "Architecture and Design of Efficient 3D Network-on-Chip (3D NoC) for Custom Multicore SoC," 2010 International Conference on Broadband, Wireless Computing, Communication and Applications, Fukuoka, 2010, pp. 67-73.

[8] M. Hamieh, M Ariaudo, S. Quintanel and Y. Louet,"Sizing of the physical layer of RF inra-chip communications," in 21st IEEE International Conference on Electronics, Circuit and Systems (ICECS), 2014, pp.163166.

[9] M-C. Frank Chang, Jason Cong, Adam Kaplan, Chunyue Liu, Mishali Naik, Jagannath Premkumar, Glenn Reinman, Eran Socher, and SaiWang Tam. 2008. "Power reduction of CMP communication networks via RF-interconnects". In Proceedings of the 41st annual IEEE/ACM International Symposium on Microarchitecture (MICRO 41). IEEE Computer Society, Washington, DC, USA, 376-387.

[10] E. Unlu et al., "An OFDMA based RF interconnect for massive multicore processors," 2014 Eighth IEEE/ACM International Symposium on Networks-on-Chip (NoCS), Ferrara, 2014, pp. 182-183.

[11] W. Hu, C. Wang and N. Bagherzadeh, "Design and Analysis of a Meshbased Wireless Network-on-Chip," 2012 20th Euromicro International Conference on Parallel, Distributed and Network-based Processing, Garching, 2012, pp. 483-490.

[12] D. DiTomaso, A. Kodi, D. Matolak, S. Kaya, S. Laha and W. Rayess, "A-WiNoC: Adaptive Wireless Network-on-Chip Architecture for Chip Multiprocessors," in IEEE Transactions on Parallel and Distributed Systems, vol. 26, no. 12, pp. 3289-3302, 1 Dec. 2015. 
[13] D. Zhao, Y. Wang, J. Li and T. Kikkawa, "Design of multi-channel wireless NoC to improve on-chip communication capacity!," Proceedings of the Fifth ACM/IEEE International Symposium, Pittsburgh, PA, 2011, pp. $177-184$.

[14] C. Wang, W. Hu and N. Bagherzadeh, "A Wireless Network-on-Chip Design for Multicore Platforms," 2011 19th International Euromicro Conference on Parallel, Distributed and Network-Based Processing, Ayia Napa, 2011, pp. 409-416.

[15] Lee, S., Tam, S., Pefkianakis, I., Lu, S., Chang, M.F., Guo, C., Reinman, G., Peng, C., Naik, M., Zhang, L., Cong, J. (2009). A scalable micro wireless interconnect structure for CMPs. MobiCom.

[16] Wu R., Wang Y., Zhao D., "Low-cost deadlock-free design of minimaltable rerouted xy-routing for irregular wireless nocs". Proc.ACM/IEEE Int. Symp. Networks-on-Chip (NOCS), May 2010, pp. 199206.

[17] R. Wu, Y. Wang and D. Zhao, "A Low-Cost Deadlock-Free Design of Minimal-Table Rerouted XY-Routing for Irregular Wireless NoCs," 2010 Fourth ACM/IEEE International Symposium on Networks-onChip, Grenoble, 2010, pp. 199-206.

[18] D. Matolak et al., "Wireless networks-on-chips: architecture, wireless channel, and devices," IEEE Wireless Commun., vol. 19, no. 5, 2012.

[19] S. Abadal, A. Mestres, J. Torrellas, E. Alarcon and A. Cabellos-Aparicio, "Medium Access Control in Wireless Network-on-Chip: A Context Analysis," in IEEE Communications Magazine, vol. 56, no. 6, pp. 172178, June 2018.

[20] F. Drillet and al., "Flexible Radio Interface for NoC RF-Interconnect," in 17th EuroMicro Conference on Digital System Design, 2014.

[21] V. Catania, A. Mineo, S. Monteleone, M. Palesi and D. Patti, "Noxim: An open, extensible and cycle-accurate network on chip simulator," 2015 IEEE 26th International Conference on Application-specific Systems, Architectures and Processors (ASAP), Toronto, ON, 2015, pp. 162-163.

[22] Wu, Yue et al. "A survey of routing algorithm for mesh Network-onChip." Frontiers of Computer Science 10 (2016): 591-601. 